\title{
Generation of Parcelation Proposals Aided by LiDAR Derived Spatial Cues
}

\author{
Sagi Filin, Aviram Burka, Yerach Doytsher \\ Technion - Israel Institute of Technology \\ Haifa, 32000, Israel
}

\begin{abstract}
Due to rapid urban development, it becomes necessary at times to document the "as made" parcelation as a means for creating the cadastre. Such documentation can involve substantial efforts, particularly when large, densely built, regions are concerned. We propose a method for generating proposed parcelation that is based on geometrical concepts of equal partitioning on one hand, and on documenting existing partitions on the other hand. We make use of Light Detection and Ranging (LiDAR) data for generating the proposed partition. LiDAR allows detecting buildings across which parcel boundaries are established, and more importantly weak cues that indicate the existence of the actual boundaries of the parcels. We show that even with modest resolution such cues can be found in the data and utilized. The wide coverage of the already georeferenced LiDAR data allows applying the proposed model on wide regions.
\end{abstract}

\section{INTRODUCTION}

Cadastre is the method of registering land, designed to ensure the rights of individuals and the state of their property. In Israel, as in many other countries, the responsibility for managing the lands is subdivided between two governmental agencies - Survey of Israel (SOI - the governmental geodetic, topographic and cadastral mapping agency) that is responsible for measuring and preparing the cadastral maps and the Land Registry Department (within the Justice Ministry) that is responsible for registration of land ownerships. A third key-player is the Interior Ministry (together with all the regional and local planning committees) which is responsible for preparing the statutory land-use programs. All these authorities are involved in ongoing cycles of parcelation and re-parcelation of land. The first cycle (initial parcelation and registration of land ownerships) is composed of measuring the lands, preparing the cadastral maps and then registration of the ownerships. All other cycles (reparcelation cycles) start by a new urban and/or regional planning, which is carried out based on the previous cadastre (previous cycle of parcelation or re-parcelation), followed by the preparation of mutation plans, updating the cadastre maps, and only then the registration phase of the new and updated ownerships of the land reparcelation. Cadastral measurement is therefore, a continuous process, due to changes in land ownership, union and division of parcels, and the constant necessity to redefine and update the ownership boundaries.

Modern cadastral mapping started in Israel in the 1920s, based on the Torrens method - Registration of Titles. This system defines the cadastral blocks (subdivision unit, usually containing 50 to 100 parcels and depicted on a single map sheet) and parcels (smallest subdivision unit) based on official surveying and mapping that is carried out (in Israel) by the state and is linked to the national coordinate network. Measurement 
results are recorded in field books, and used to determine the boundaries of the blocks and the parcels, as well as other features (buildings, fences, electric poles, etc.). All these measurements are depicted graphically on field plan sheets. Maps of the cadastral blocks are prepared based on the field sheet, consisting of all parcels in the block and all included features. These cadastral maps contain neither the measured data nor any dimensions whatsoever of the parcel boundaries and serve only as a graphical presentation of parcel layout. The field books and field sheets are the evidence for the statutory validity for the cadastral subdivision, and when it is necessary to restore the cadastral boundaries, this existing information in the field books and sheets, is being used in relation to nationwide control network to restore the boundaries (Shoshani et al., 2004).

This organized mechanism of urban/regional planning which is followed by preparing mutation plans, updating the cadastre maps and registering the updated ownerships of the lands, is disturbed when informal construction is carried out - informal in terms of non-legal, not authorized or without approved/official land-use plans. In Israel, as in many other countries, this informal construction is carried out only in two extreme situations. The first situation is when private citizens bypass the regulations and the land-use formalism, and build their homes without any legal permission and occasionally even not on their own land. The second situation is when the state itself (e.g. governmental agencies or ministries) decide to bypass its own law and its regulations and build residential neighborhoods on unplanned and without approved land-use plans. This kind of governmental violation of the construction and housing laws and regulations occurred first during the 1950s and then again during the late 1980s and beginning of the 1990s. The first time was immediately after the establishment of the state of Israel, when within a very short period of time a very huge wave of new immigrants arrived to Israel and during one decade the population of the country almost tripled. There was no time, nor skilled manpower to start an organized process of urban planning, cadastral surveying and then land ownership registration. The practical solution was first to build houses for all those hundred thousands immigrants, and only then to find the legal solutions for surveying, parcelation and registration of land ownerships. The second time when there was a necessity to a governmental bypassing of construction laws and regulations happened about 15 years ago, when during a short period of 3-4 years almost one million immigrants came to Israel from Russia.

In all these informal construction cases, there is a need to legalize the illegalized houses and neighborhoods. This is carried out by measuring the as-made built-up areas, suggesting a posteriori sub-division of the land, preparing mutation plans that depict the actual land-use situation, and finally preparing the cadastral maps and registering the ownerships. In the process of measuring the as-made built-up areas the surveyors will map the buildings as well as fences, walls etc. in order to be able to suggest a "reasonable" subdivision of the area (between and around the existing houses). This task can be time consuming and expensive, especially when performed on large scale. In this paper, we study models for autonomous generation of a first parcelation proposals based on spatial cues. The proposed model has the advantage of providing a first parcelation sketch for large regions and with little intervention by an operator. The spatial cues that are proposed here are in the form of buildings but also on peripheral weaker cues that define the parcel boundary location. Sensory 
information in the form of Light Detection and Ranging (LiDAR) data and images are being used for this end.

\section{PARCELATION MODEL}

Delineation of parcel boundaries can be a laborious if preformed manually either via photogrammetry and even more so via ground surveying. This work becomes even more involved in built-up areas, which are cluttered and not accessible at times. In many cases, particularly in urban environments, it is expected that spatial "markers" will delineate the parcel boundary. Such markers can relate to borderlines, like fences that surround buildings, or breaklines between the ground level of buildings and the street. If recognizable, such objects can be used to generate a partitioning that often reflects the actual (or eventual) parcelation of cadastral blocks. We explore the level of automation that can be reached in generating parcelation proposals from remotely sensed data. This first partition that document "facts" on the ground can then be further refined by a human expert that will add the awareness to nuances and the actual limitation and regulations that should be imposed.
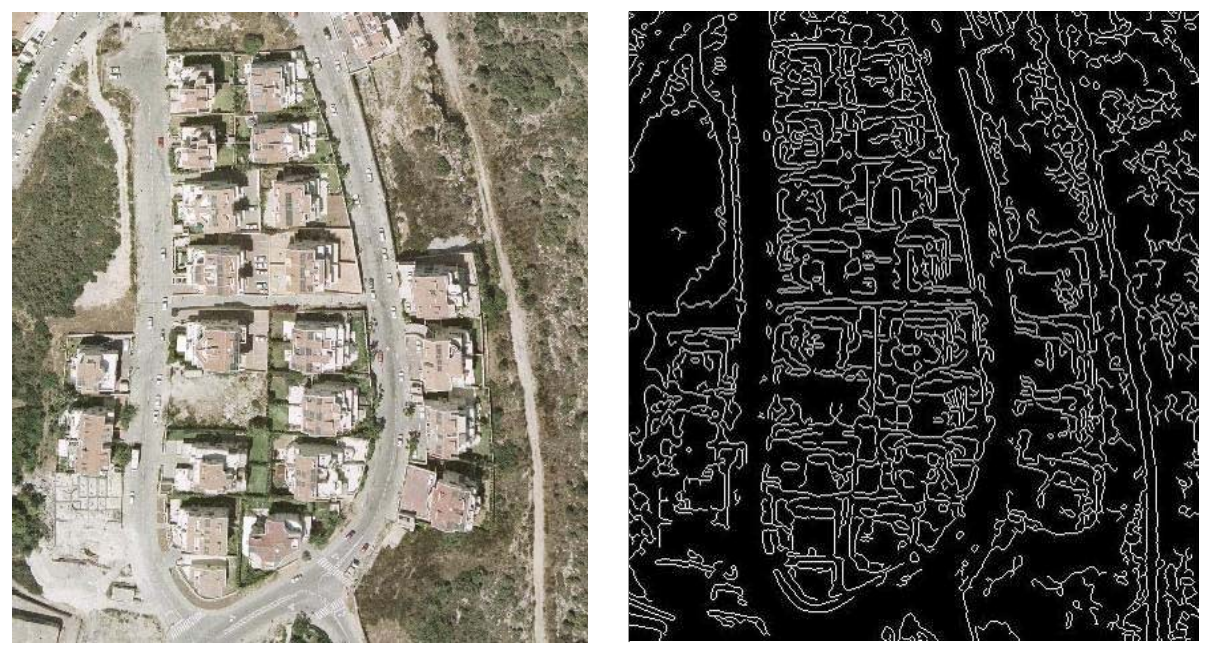

Figure 1. An orthophoto map of a typical residential area (left) and its resulting edge map (right)

Among the candidate sensory data, images enjoy both high spatial resolution and high spatial accuracy. Figure 1 shows an orthophoto of a residential neighborhood with two rows of buildings and fences surrounding them. The general partitioning is relatively clear, but when translating this visual impression into autonomous imagebased analysis the limitations of such approach can be readily seen. Using wellknown machine-vision procedures (e.g., edge detection or image segmentation) the results clearly show that intensity edge-based data do not necessarily (and in fact, hardly) reflect physical boundaries between real world objects (see e.g. Castleman, 1996). Essentially, image edges reflect discontinuity in intensity, which only oftentimes reflect the outline of physical objects. Sorting out the edges that define objects from the complete edge map is practically impossible. As a consequence, we turn to airborne laser scanning data as the primary source of information for the generation of parcelation proposals. In recent years, LiDAR technology became a dominant tool for mapping the topography in large scale. By documenting in high resolution and in $3 \mathrm{D}$ the terrain and objects on the terrain, LiDAR data facilitate a 
detailed georeferenced mapping of spatial objects. Accuracy wise, a growing number of publications that report accuracy level on order of $\pm 10-20 \mathrm{~cm}$, in elevation and position.

The choice of LiDAR data is not only driven by the ability to produce a detailed and accurate description of the topography. Compared to the standard image based mapping, the detection and characterization of geographic objects that have distinct topographic signatures is greatly enhanced with data that capture the actual height information. As such the application of LiDAR data for building reconstruction, tree and forest modeling echo this ability. Whereas buildings or trees are dominant objects compared to their surrounding, natural boundaries, as those we seek, are relatively small (in fact narrow) in size, and not extremely distinguishable in large datasets. Their detection therefore deviates from the standard procedures and poses a challenge, particularly if autonomous detection is of concern. Solving this, our proposed model approaches the detection of boundary points indirectly by searching first for a distinct anchor objects and then using them for a subsequent search of border lines. To strengthen the reconstruction model we consider also the fusion of LiDAR data and image based data as a means to further enriching the boundary information. As an outline the model we propose consists of the following steps, listed in Table 1

\section{LiDAR based parcelation model}

1. Detection of anchor objects.

2. Generation of first partition.

3. Search for subtle spatial cues both in the laser data and in images to reinforce the partitioning.

4. Fusion of both boundary data sources within an adjustment process as a proposal for the parcelation.

5. Finalization of the parcelation proposal based on the adjusted information.

Table 1. Outline of the proposed parcel boundary reconstruction algorithm

In the following sections, we describe in detail the model applied in each of the individual steps.

\subsection{Autonomous extraction of anchor objects}

Anchor objects should be both distinguishable and usable in locating the spatial features that refer to the boundary objects. As anchor objects we use buildings. Our choice is motivated by the fact that buildings are both distinct objects compared to their surrounding and therefore can reliably be detected, and since parcels are usually formed around them they provide an initial cue for the location of the parcel. Detection of buildings in laser data has been researched quite extensively in the past (see e.g., Vosselman and Dijkman, 2001; Rottensteiner and Briese, 2002; Abo-Akel et al., 2005) and is likely to remain so in the near future. The approach we take for 
autonomous detection of buildings begins with the object-to-background separation concept, with the background being the terrain. Having the terrain identified and removed from the laser point cloud, the detached objects appear as isolated laser patches. These patches become the target of a subsequent analysis that aims identifying the actual buildings from the rest of the non-terrain objects.

Our object to background separation strategy is driven by a filtering approach that uses global functions in the form of orthogonal polynomials for a coarse separation between the terrain and the detached objects and is followed by surface refinement (see Abo-Akel et al., 2007 for greater detail). The polynomial coefficients are estimated robustly in an iterative process with a guiding assumption that when a function is fitted to a mixture of terrain and off-terrain points, off-terrain points will have positive residuals while terrain points will have negative ones. To reduce the effect of off-terrain points on the fitted function, the weight of points with a positive residual is reduced between iterations, thereby reducing their influence. Within the iterations cycle, the shape of the polynomial is simplified by a decrease of its degree; the controlled shape of the function, coupled with the reduced influence of the offterrain points limits further the influence of the latter while offering a closer description of the terrain. At the final iteration the shape of the terrain does not change, namely, off-terrain points are not influencing the shape of the polynomial and only the terrain points influence the results.

Having the terrain point identified and isolated, the detached points are grouped into segments. Those segments are further filtered by eliminating segments that are too small to be considered as buildings (namely area too small to form a building). On the remaining ones a height difference based analysis is applied. Object heights are compared to their surrounding and objects whose relative height exceeds a prescribed height (usually $d_{h}>3 \mathrm{~m}$ ) are considered building candidates. ${ }^{1}$ Experiments show however that sometimes a single detached object is in fact an elevated level above the bare earth that consist of more than one building. Therefore height differences are tested between detached objects and the surrounding terrain points as well as within the detached objects. Regions whose height difference exceeds a prescribed value are considered building boundary points.

\subsection{Computation of the first proposal for parcelation}

For the generating parcelation proposal, reconstruction of building shapes is less of an interest here. Our main concern is the delineation of the parcel boundaries surrounding them. Since parcel boundaries do separate the regions between buildings, we use a Voronoi based separation as an initial step for establishing this partition. The Voronoi partitioning into cells is defined according to the following criterion

$$
\mathrm{v}\left(p_{i}\right)=\left\{q|| p_{i} q|<| p_{j} q \mid \quad j \neq i\right\}
$$

with $p_{i}, p_{j}$ the set of points in space (some times termed sites) and $\mathrm{q}$ the set of points in spaces that are closest to $p_{i}$ than to any other site. The set of points, $q$, define the

\footnotetext{
${ }^{1}$ We note that more elaborate methods for differentiating buildings from their surrounding exist, but for our application were not particularly needed.
} 
Voronoi cell for each site and the equidistant points provide the Voronoi diagrams. Building centroids are defined as the Voronoi sites. Geometrically the Voronoi partitioning will be the basis for the optimal parcel boundaries.

Handling boundary conditions- one feature of the Voronoi diagram which has undesired consequences is the open Voronoi cells for boundary points, those forming the convex hull. Since unbounded regions do not exist in cadastral data we devise a simple method for bounding them. For doing so we mirror points neighboring the boundary points along the line connecting them. We then recomputed the Voronoi diagram and throw away the duplicated points. Mirroring the points ensures that the original points will have closed cells with distance equivalent to their distance to the inner neighboring points.

It is noted that Voronoi diagrams have been proposed as a means for space partitioning for higher dimensional features such as lines (in 2D and 3D) and polygonal objects. While the application of a polygonal based Voronoi based partitioning seem at first a more appropriate solution, we note that building boundaries may oftentimes include concavities (consider e.g., an L shape building) that may in turn result in concave boundaries around the object. Realizing the building contour that may be as complex as desired should not have direct influence on the parcel boundary shape we adhere to the centroid-based point-based Voronoi diagram for the generation of the first partitioning sketch.

\subsection{Detection of spatial cues}

The Voronoi space partitioning is founded on solid geometric reasoning (equal space cell partitioning). However it has no physical evidence to consolidate it. Spatial objects in the data that form "obstacles" (e.g., fences and breaklines) document spatial discontinuity that usually reflects "natural" boundaries. Under the assumption that objects with height signature should be noticeable to their surrounding we turn to search for spatial cues that echo the existence of border objects. The sought after objects do not enjoy the sharp object to background contrast that buildings have. However, in the current case the search for them is much narrower since the Voronoi diagram already offers a first sketch; our search is therefore limited to the surrounding of the Voronoi lines. The detection of spatial border related cues is therefore implemented as follows:

1. For each Voronoi line, whose parameters are $\left\{A_{j}, B_{j}, C_{j}\right\}$ in implicit form, find the laser point set $p$, within a prescribe distance to the line, namely

$$
p=p_{i} \mid d \leq \frac{A_{j} x_{i}+B_{j} y_{i}+C_{j}}{\sqrt{A_{j}^{2}+B_{j}^{2}}}
$$

with $d$, the distance criterion, the point set fulfilling the distance criterion, $p_{i}=\left\{x_{i}, y_{i}\right\}$ a candidate point.

2. For each point within the point set, establish the neighborhood surrounding it, and compute the first derivative. 
3. If the first derivative exceed a prescribe value that designate the minimal obstacle height compared to the background, consider the point as edge points.

The outcome is a set of laser boundary points. Following the extraction of the laser boundary points we turn to improve our model by adding image based information.

Extraction of image based information - Since images are a far available source, we consider their contribution to the parcel localization process. The information we make use of arrives from analyzing discontinuities in the intensity. Such discontinuities (termed also edges), are detected by studying the steepness in transition between intensity values of neighboring pixels (gradients), and result in an edge map that document those gradients whose strength exceed a threshold values. While inferring from those edges about the existence of buildings and their locus is a hard task, performing an informed search along the Voronoi diagram lines for high intensity response, is doable. Edges (in fact edge pixels) that fall within the predefined search buffer are considered as further information for the localization of the parcel boundary.

\subsection{Fusion and computation of the parcelation}

The main objective of fusing the intensity based parcels boundaries and the laser related ones is approximating the parcel boundary. The result should therefore be in the form of a line that best suits both data sources.

Since the line we seek should best fit information from both data sources we choose a least-squares solution that has the desirable property of minimizing the $l_{2}$ norm between observations. The general framework for the adjustment will consider information from two different populations, i) laser points with stochastic properties of $p_{l} \sim\left(0, \sigma_{l}\right)$ and, ii) image based edge points whose stochastic properties are $p_{i} \sim\left(0, \sigma_{i}\right)$. The line equation is then solved by linear regression

$$
\hat{\xi}=\left(A^{T} P A\right)^{-1} A^{T} P y
$$

with $\hat{\xi}$, the adjusted parameters, $A$ the design matrix containing the observation equations, and $y$ the observations. ${ }^{2} P$, the weight matrix is composed of relative weights to the image based points and laser related ones. Those are primarily derived from the spatial resolution of both data sources.

As a final step we readjust the parcel corners. Of particular relevance are those corners that join three lines or more. The adjustment of the corners (which are also the end points of the parcel border lines) can be viewed as the adjustment of the end points for all lines meeting at the same corner. In this form the observation equations will be in the form

${ }^{2}$ We note the sensitivity of the adjustment of lines in explicit form to their orientation, the degeneracy of line adjustment in the implicit form, and the limitation of simple regression in minimizing discrepancies along only one direction. Solutions that account for all these problems are fairly 
standard and therefore not discussed here further. 


$$
C_{i}=A_{i} x+B_{i} y \quad i=1, \mathbf{K}, n
$$

where $A_{i}, B_{i}, C_{i}$ are the line parameters for line $i, x, y$ are the adjusted end point coordinates, and $n$ the number of lines joining at the adjusted end point. Following the adjustment of all end points, which is carried out sequentially, the parcelation proposal, is completed. For the adjustment of the parcel's end-points we can integrate our level of confidence in the border line as resulted from their adjustment $\left(V_{\hat{\xi}}=\hat{\sigma}_{0}\left(A^{T} P A\right)^{-1}\right)$. This way, lines that are better defined will receive a proper weight. We also point to the possibility of performing a simultaneous adjustment of all end points based on the raw image and laser derived information. The choice of the approach we took lies in its simplicity, as being sequential by nature. For the cost of a slight lose in accuracy that the simultaneous adjustment offers, we enjoy a more controlled set of computations that can be performed sequentially.

\section{EXPERIMENTAL RESULTS}

The dataset for testing the proposed model is composed of a LiDAR data with point density of $\left(\sim 0.25\right.$ point $\left./ \mathrm{m}^{2}\right)$, and an orthophoto of the same area. Figure 1 shows the orthophoto of the area, and in Figure 2 a shaded relief map of the region can be seen as well as the actual 3D point cloud of the region.
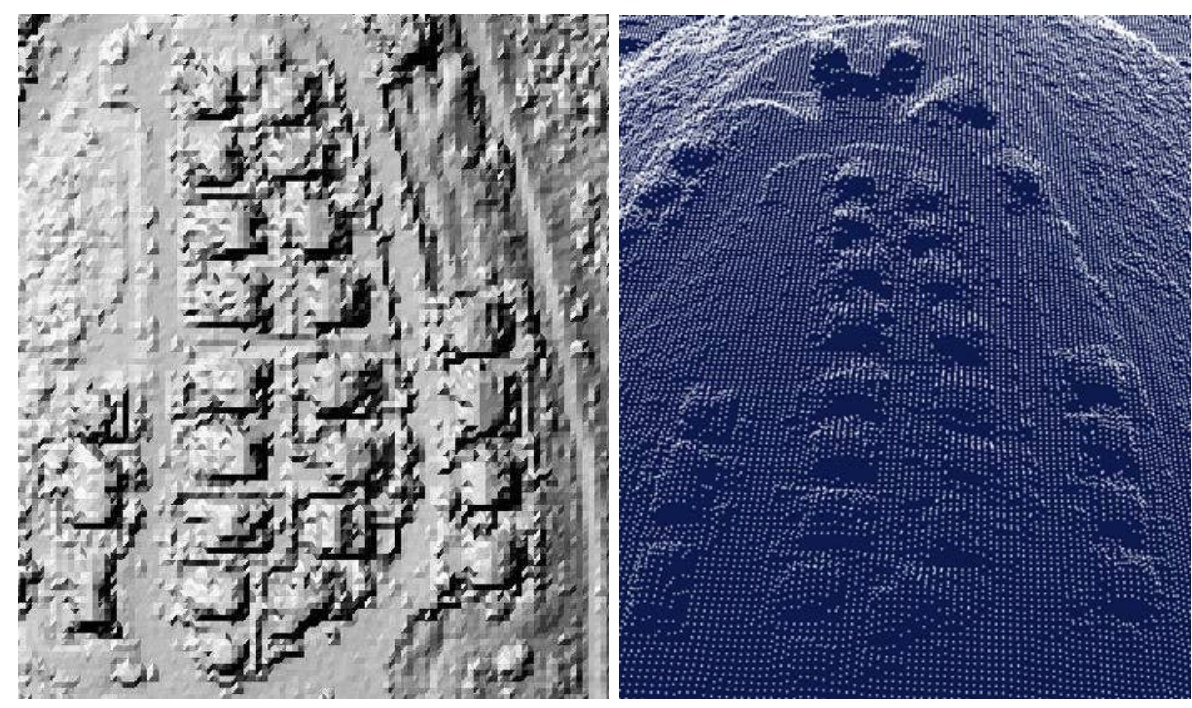

Figure 2. Airborne laser data in urban region - shaded relief map (left), and the raw point cloud (right)

As Figure 2 shows even in a complex topography as the one presented the buildings stand out quite clearly compared to their background. The comparison between the left and right sub-figures shows the intricate nature of the boundary lines in the laser data. While they are visually noticeable in the shaded relief map, in the original data their signature is minor compared to their surrounding. Generally, with higher resolution data physical obstacles such as fences and walls will receive a much stronger response. This can be clearly shown in Figure 3 that features laser scanning data with higher resolution (4 points $/ \mathrm{m}^{2}$ ). The fences (vertical object on the ground that appear in dark gray tone) were detected here directly with no need to define 
anchor objects around them using segmentation procedures defined for 3D laser point clouds.

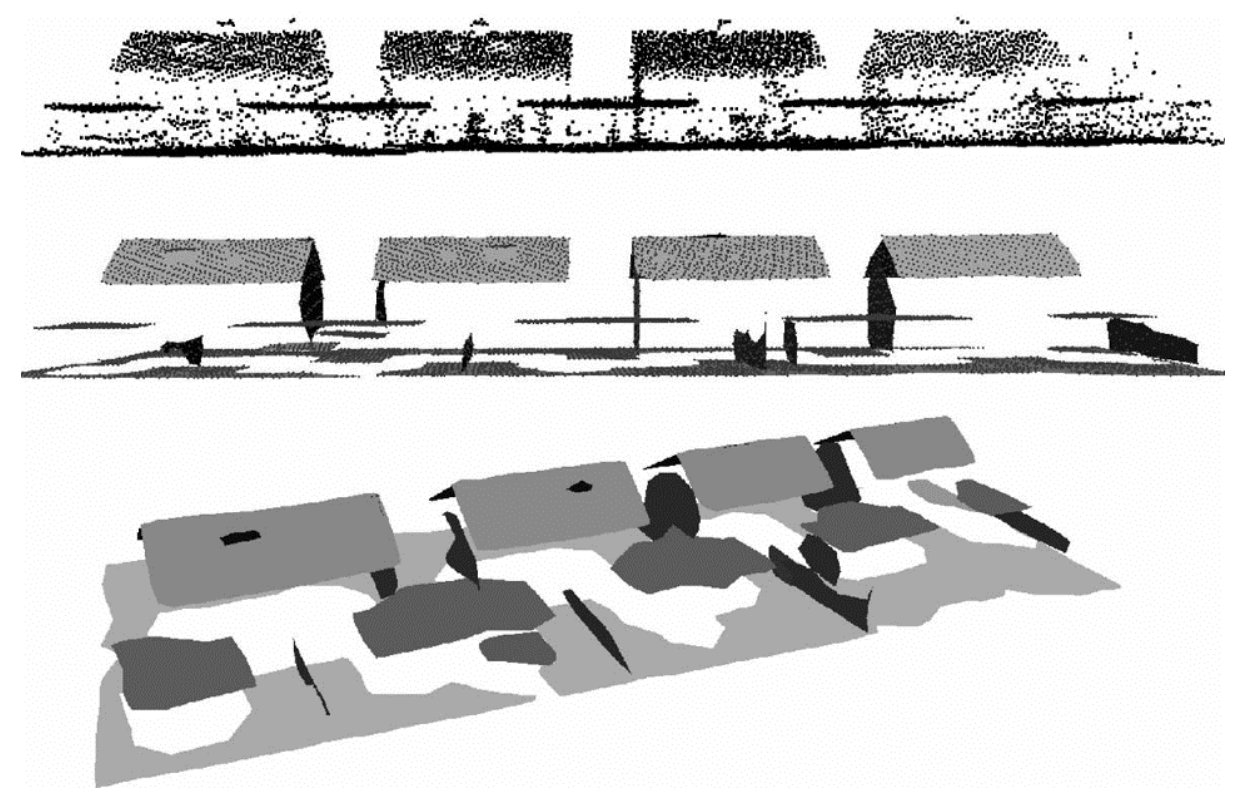

Figure 3. Vertical structures in high density LiDAR data - the original point cloud (top), the point cloud following segmentation (middle), and a side looking view of the segmented dataset that show the realization of the fences in the data (down)

Using the data with the lower resolution, the first stage is separating the detached objects from the terrain, using the filtering algorithm that was describe in Section 2.1. Results of the filtering can be seen in Figure 4

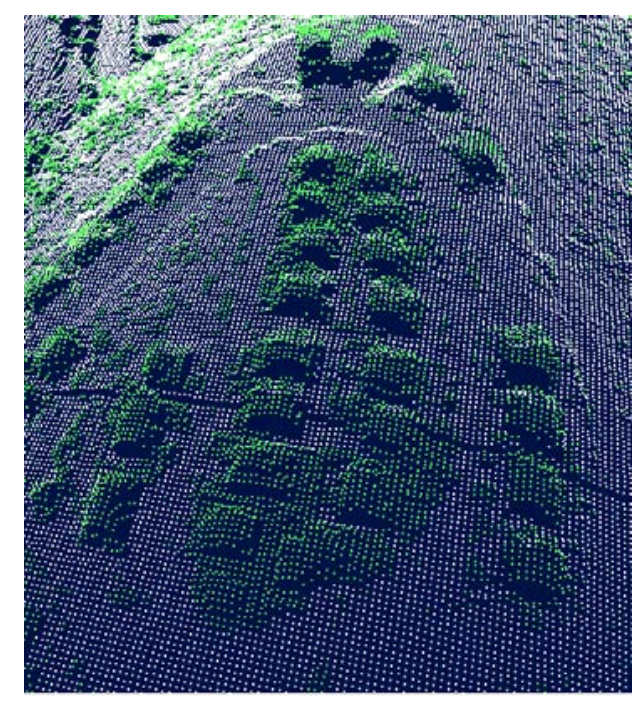

Figure 4. Separation of object from the background using filtering - white points are the terrain, green detached objects

The filtering algorithm has managed separating the detached objects from the terrain. The results show indeed that buildings are now well defined objects compared to their surrounding. On the slopes of the hill one can see vegetation patches that will be filtered out due to their size and relative height. In the front one can notice that five of the buildings are located on ground whose level is detached from the terrain. As was 
noted earlier, considering this all region as one potential building will lead to a wrong detection (resulting in detection of one mega building). We therefore analyze the candidate detached patches in reference to their surrounding as well as within themselves.

The analysis is being carried out by analyzing elevation gradients of these objects. Strong gradients indicate sharp height variation, and therefore potential building boundary. By tracing the gradients to form a close polygon we delineate the building boundary. Figure 5 shows the detected buildings following their detection and centroid computation.
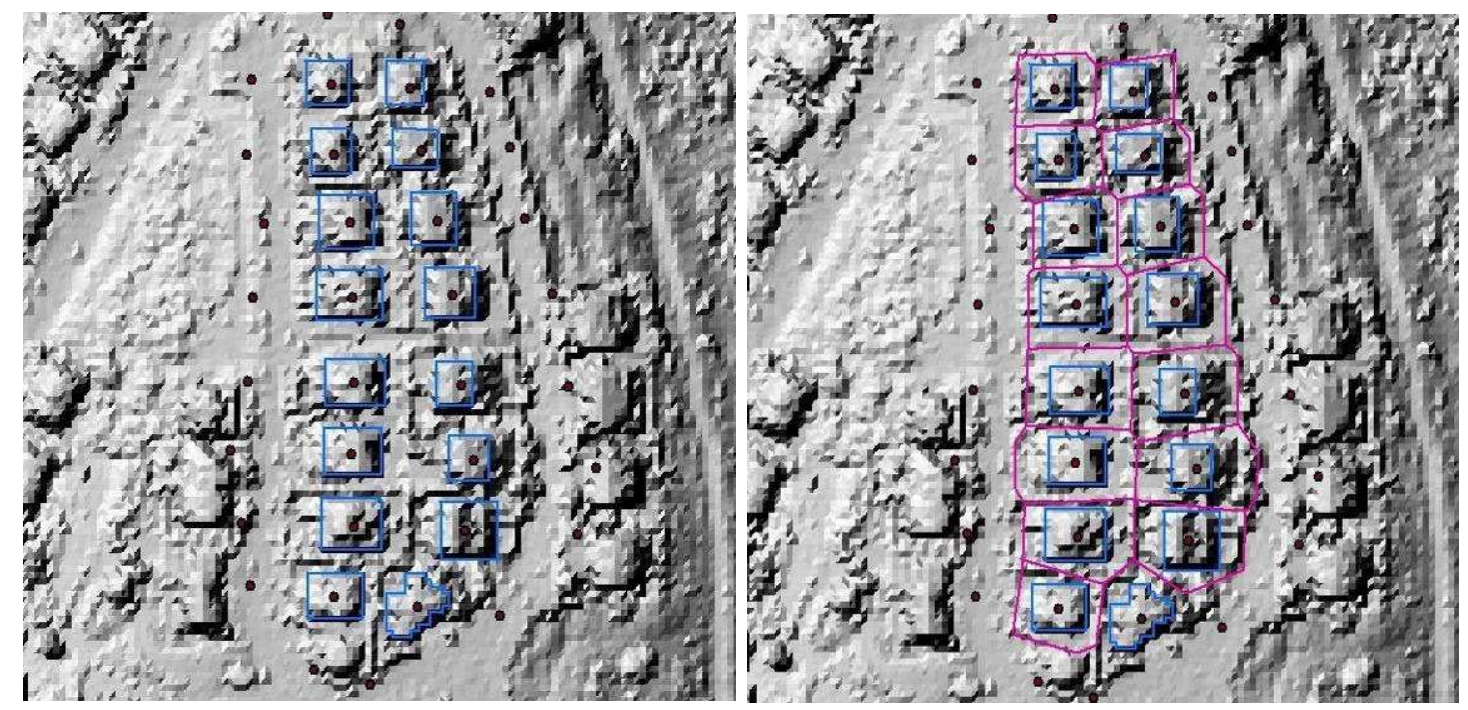

Figure 5. The detection of the building (left), and the resulting first parcelation sketch (right)

As can be seen, all buildings were correctly detected and their centroid indeed reflects their placement in space. The boundaries (generalized where possible into bounding squares) are sufficient to represent their shape (considering their resolution, our application, and original shape). Compared to the image based edges, where buildings could hardly (if at all) be detected, the LiDAR based autonomous detection only shows its strength and great potential for mapping purposed of various kinds. In Figure 5 (right) the first proposal for Voronoi diagram can be seen. One can see that the Voronoi lines appear not far from the actual location of the actual separating fences. Outside our region of interest one can see a few sporadic points; these artificial mirror points help solving the open cells of the outer points in the Voronoi diagram.

Following the generation of the first parcelation sketch we now seek physical evidence in the form of discontinuity in heights in the data. Using the first sketch derived by the Voronoi diagram the search for fence points (again consider their actual appearance in the data as isolated points) is now localized, and with this the ability to search for subtle height variations (e.g., $\sim 1 \mathrm{~m}$ height difference of a potential point compared to its surrounding). Results of this search are featured in Figure 6 where the green points are the laser fence related points. 


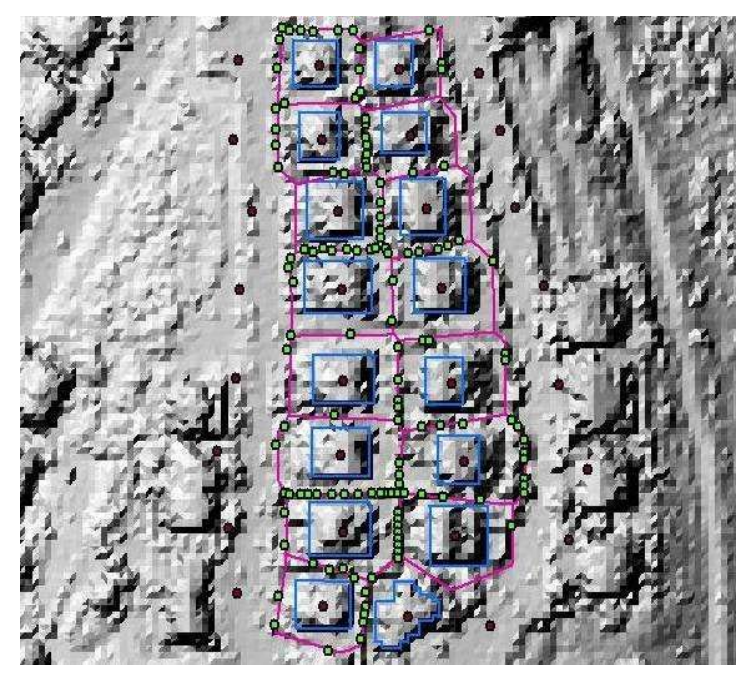

Figure 6. The detected laser points
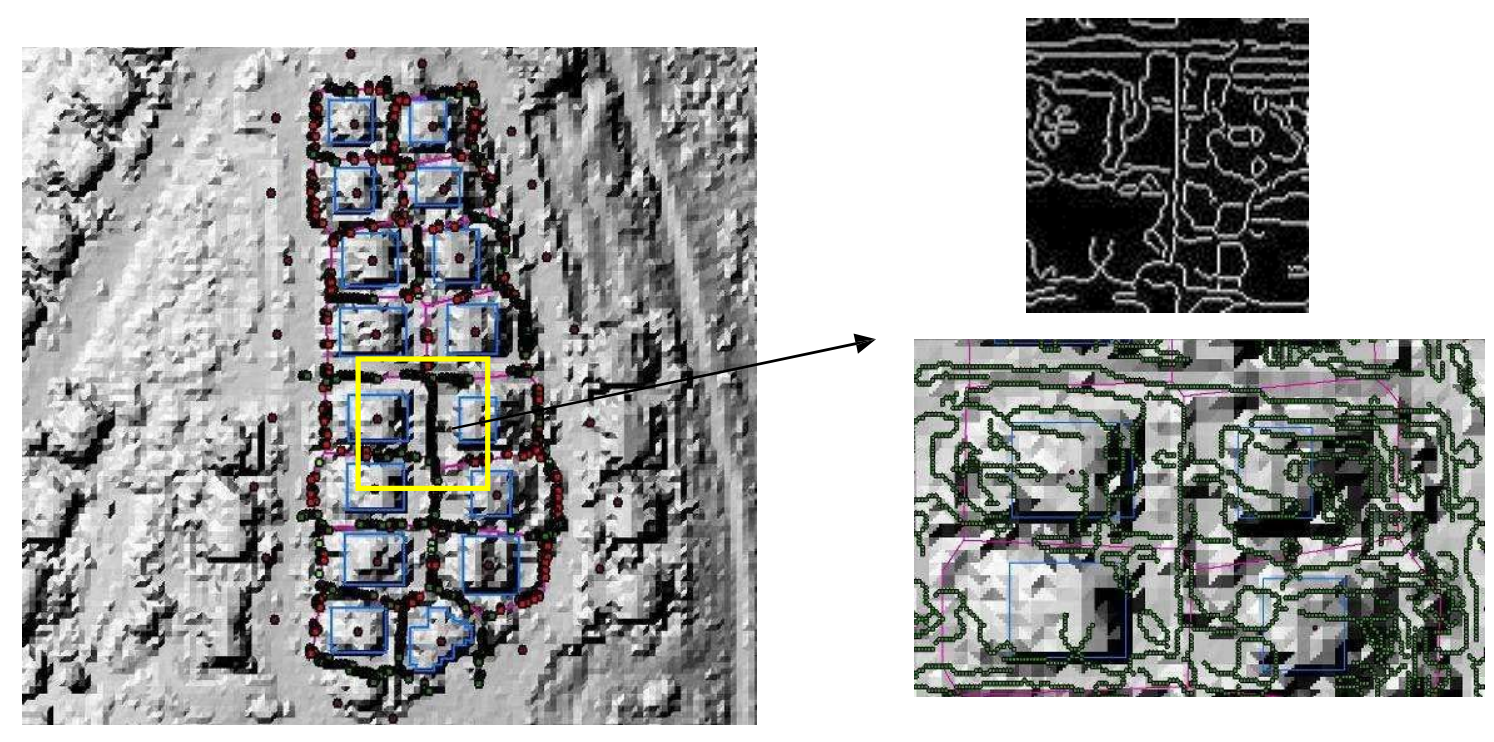

Figure 7. Combination of LiDAR and intensity edges (left), and the blowup of the original edge map from the intensity images (right)

The inclusion of intensity based edge points is illustrated in Figure 7. The evident fact shown in Figure 7 is that the number of image based edge-points is far greater than those arriving from the sparse laser data. Additionally, edge points were detected where no laser edges can be seen. This abundance of information should be attributed to the superior spatial resolution of the image based data, and information encapsulated in the intensity data. Nonetheless, without the support of the LiDAR based analysis their detection could not have been materialized. This result indicate the great merit in fusing both sources of information where low resolution but rich in information LiDAR data coupled with intensity data make up for the shortcomings of the individual sources. Returning to Figure 1, one can be seen that edges that reflect the existence of border lines are present. However, when applying the same procedure as the one we applied for the LiDAR edges, the outcome is both edges that reflect the actual fences and non-related ones that reflect mere discontinuities in intensity (see blowup in Figure 7). Since the inclusion of edges points is based on buffer operations,

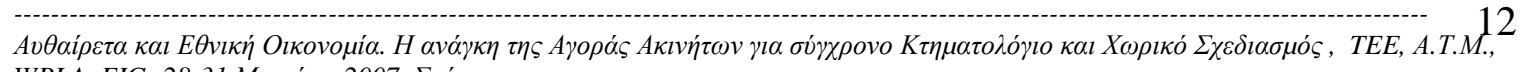

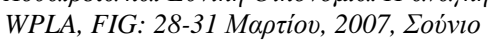


the non-related edge points document, in fact, the buffer and thereby the Voronoi property. This contrasts our general objective of documenting the actual physical boundaries. To alleviate this, we analyze the candidate edge pixels not only by their inclusion but also by their orientation (orthogonal to the gradient direction). With the orientation criterion applied, only edge pixels arriving from "fence" related edges will remain.

Following the detection of the physical related boundary lines the adjustment procedures for the individual lines and their end-points follows to complete the computation of the parcelation proposal.

\subsection{Analysis and outlook}

The result following the adjustment can be seen in Figure 8. Analysis of the resulting edges shows that offsets on order of $1 \mathrm{~m}$ from the Voronoi boundaries are detected. From Figure 8 one can see that the boundaries along follows the parcel boundaries much tightly than the original Voronoi based proposal. In fact where edge information exists, the boundary lines have adapted their shape.

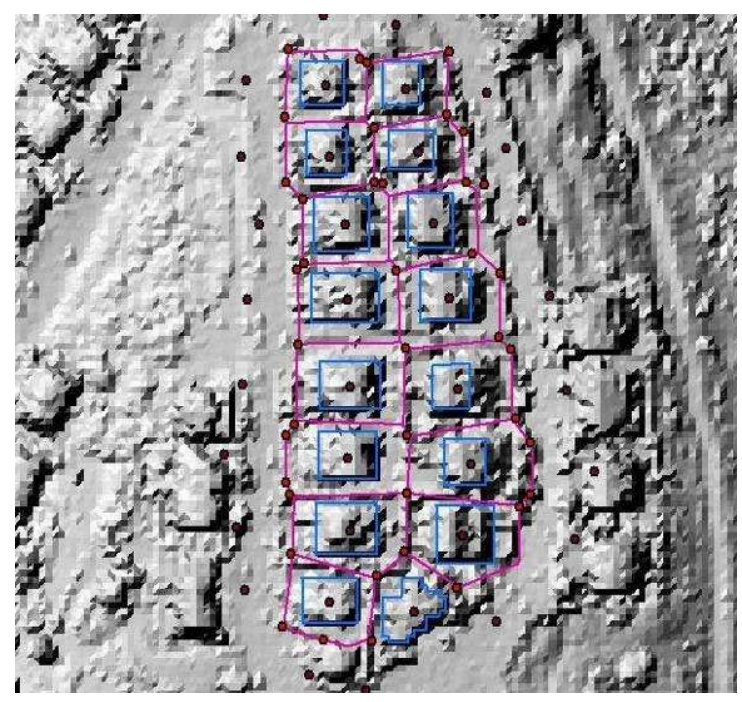

Figure 8. The results following the adjustment of the parcels boundaries

The results show the great potential of LiDAR based interpretation for urban region and validate our original hypothesis that by a proper utilization of remotely sensed data, parcelation proposals can be generated autonomously. Future development of the proposed model will look into the enforcement of additional geometric constrains like perpendicularity and parallelism between lines, relaxation of the edge orientations to avoid being overly confined by the lines' orientation as dictated by the Voronoi proposal, as well as extending our use of spatial information, e.g., roads as parcel delimiters. Overall, the proposed parcelation reflects the actual partition and echo the existence of physical boundaries within the data. Considering the fact that such partition was form autonomously only foster the logic in further pursuing such approach for the registration of informal settlements on a large scale. 


\section{References}

Abo Akel N., Filin S., Doytsher Y., 2007. Orthogonal Polynomials Supported by Region Growing Segmentation for the Extraction of Terrain from LiDAR Data. PE\&RS (in press).

Abo Akel N., Filin S., Doytsher Y., 2005. From Airborne Laser Data to Spatial Information: Object Reconstruction and Accuracy Analysis. Proceedings of FIG working week 2005 and GSDI-8. Cairo, Egypt, April 16-21.

Castleman K. R., 1996. Digital Image Processing. Prentice Hall, Englewood Cliff, New Jersy, 667 p.

Rottensteiner F., Briese C., 2002. A New Method for Building Extraction in Urban Areas from High Resolution LiDAR Data. International Archives of Photogrammetry and Remote Sensing, 34(3A): 295-301.

Shoshani U., Benhamu M., Goshen E., Shaul Denekamp S., Bar R., 2004. Registration of Cadastral Spatial Rights in Israel - A Research and Development Project, Proceedings of FIG working week 2005, Athens, Greece, May 22-27.

Vosselman G., Dijkman S., 2001. 3D Building Model Reconstruction from Point Clouds and Ground Plans. International Archives of Photogrammetry and Remote Sensing, 34(3/W4): 37-43. 